\title{
Molecular dynamics simulation of the regrowth of nanometric multigate Si devices
}

\author{
Luis A. Marqués, ${ }^{1, a)}$ Lourdes Pelaz, ${ }^{1}$ Iván Santos, ${ }^{1}$ Pedro López, ${ }^{1}$ and Ray Duffy ${ }^{2}$ \\ ${ }^{1}$ Departamento de Electrónica, Universidad de Valladolid, E.T.S.I. de Telecomunicación, 47011 Valladolid, \\ Spain \\ ${ }^{2}$ Tyndall National Institute, University College Cork, Lee Maltings, Cork, Ireland
}

(Received 1 September 2011; accepted 24 December 2011; published online 2 February 2012)

\begin{abstract}
We use molecular dynamics (MD) simulation techniques to study the regrowth of nanometric multigate Si devices, such as fins and nanowires, surrounded by free surfaces and interfaces with amorphous material. Our results indicate that atoms in amorphous regions close to lateral free surfaces or interfaces rearrange at a slower rate compared to those in bulk due to the discontinuity of the lateral crystalline template. Consequently, the recrystallization front which advances faster in the device center than at the interfaces adopts new orientations. Regrowth then proceeds depending on the particular orientation of the new amorphous/crystal interfaces. In the particular case of $\langle 110\rangle$ oriented fins, the new amorphous/crystal interfaces are aligned along the $\langle 111\rangle$ direction, which produces frequent twining during further regrowth. Based on our simulation results, we propose alternatives to overcome this defected recrystallization in multigate structures: device orientation along $\langle 100\rangle$ to prevent the formation of limiting $\{111\}$ amorphous/crystal interfaces and presence of a crystalline seed along the device body to favor regrowth perpendicular to the lateral surfaces/interfaces rather than parallel to them. (C) 2012 American Institute of Physics. [doi:10.1063/1.3679126]
\end{abstract}

\section{INTRODUCTION}

The demanding requirements for next generation integrated circuits impose the search for alternatives to the traditional planar field-effect transistors (FETs). Multigate metal-oxide-semiconductor devices, such as FinFETs and nanowires, are promising scaling approaches for future technology nodes due to excellent short channel effect and leakage control. ${ }^{1-4}$ These advantages translate to lower power consumption and enhanced device performance.

For junction formation, ion implantation is the leading candidate as the means to introduce dopants, as it is a well established technique and allows a good control of the resulting profiles. Amorphization of the implanted layer is often a side effect associated to the accumulation of damage caused by implanted ions. In the case of planar devices, subsequent annealing produces the complete regrowth of the amorphous region up to the $\mathrm{Si}$ surface, with a good crystalline quality and high dopant activation level in the regrown layer. Only some defects remain beyond the initial amorphous/crystal (a/c) interface.$^{5-7}$ However, in the case of nanometric multigate devices, regrowth is not as straightforward as in bulk $\mathrm{Si}$ due to the presence of lateral free surfaces or interfaces with amorphous $\mathrm{SiO}_{2}$ (see Fig. 1). ${ }^{8-10}$ They may alter the recrystallization process because regrowth occurs parallel to them rather than terminating at a surface/interface, as is the case of planar devices. ${ }^{11}$ In fact, experiments show that in narrow planar ultra-thin silicon-on-insulator devices and FinFETs, regrowth is imperfect and line defects starting at the lateral surfaces/interfaces are formed in the implanted regions, and

${ }^{a)}$ Electronic mail: 1marques@ele.uva.es. polycrystalline material is nucleated beyond..$^{9,12}$ These regrowth features, which are more acute in narrower structures, are serious concerns because of the degradation of device performance and variability. ${ }^{13,14}$

In a previous work, we showed that the atomistic simulation technique known as MD was able to reproduce the features observed in experiments. ${ }^{15}$ These results were recently confirmed by other authors also using MD techniques. ${ }^{16}$ This defected regrowth of narrow fin structures has even been modeled in a kinetic Monte Carlo code. ${ }^{17}$ In spite of all these simulation studies able to reproduce the experimental observations, no physical basis behind the defective regrowth has been given, especially regarding the role of the lateral surfaces/interfaces. Consequently, no alternatives to overcome this technological problem have yet been proposed.

In this work, we use classical MD simulation techniques to study the recrystallization behavior of narrow $\mathrm{Si}$ 3D structures (fins and nanowires) in different crystal orientations, in order to explain the experimental observations and to propose technological alternatives to overcome the defected regrowth.

\section{SIMULATION DETAILS}

Classical MD consists of the numerical resolution of the Newton equations of motion for the set of particles that constitute the system under study. ${ }^{18}$ The outcome of the MD simulation are the trajectories followed by all the particles along time, which makes this technique very adequate to simulate recrystallization processes. In our study, we have used the Tersoff interactomic potential within its third parametrization (T3), ${ }^{19}$ to describe particle interactions. T3 has been shown to accurately reproduce the properties of both amorphous and crystal phases of bulk $\mathrm{Si}^{20,21}$ and to correctly describe the 


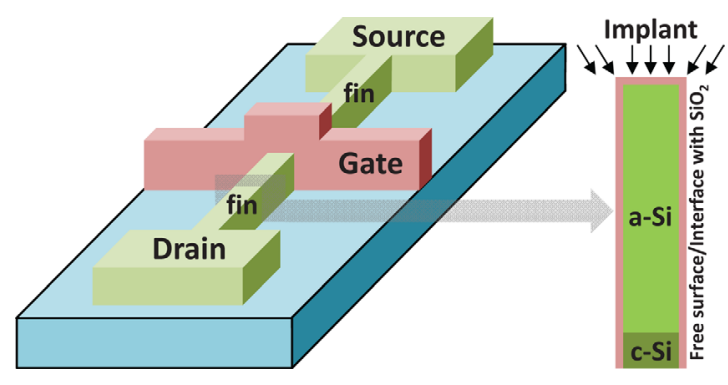

FIG. 1. (Color online) Scheme of a FinFET device. On the right, a transversal section of the fin is shown. Usually, implants required for doping amorphize the fin body but for a small region at the bottom. The inherent 3D structure introduces lateral free surfaces/interfaces which make regrowth difficult during the subsequent annealing step.

solid-phase epitaxial recrystallization from a/c interfaces in different orientations, ${ }^{7}$ which are key features for the present study. A limitation attributed to the T3 potential is that it predicts a melting point temperature for Si of around $2400 \mathrm{~K},{ }^{22}$ well above the value found in the experiments, $1685 \mathrm{~K}^{23}$ This is not a serious drawback since it is possible to make a rescaling between real and T3 temperatures. ${ }^{24}$

To study the recrystallization of narrow fin and nanowire structures, we have generated several Si samples with different crystal orientations and sizes. Initially, the samples are fully crystalline, then selected regions of them are rendered amorphous by the introduction of bond defects, ${ }^{22}$ leaving just a crystal seed at the bottom. The bond defect, first studied using tight-binding and first-principle techniques, ${ }^{25,26}$ consists of a local rearrangement of bonds with no excess or deficit of atoms, as shown in Fig. 2. Although atoms remain fourfold coordinated, the bond defect introduces disorder in the lattice since two atoms are displaced with respect to perfect lattice positions, and five- and seven-membered rings typical of the amorphous phase are created. In fact, this defect is considered as the basic building block of the amorphous phase. ${ }^{27}$ This method of introducing damage in the Si lattice is similar to the algorithm developed by Wooten et al., ${ }^{28}$ although the atomic displacements are somewhat different. The atomistic model for ion-beam amorphization and recrystallization in $\mathrm{Si}$ based on the bond defect has proved to account for most experimental observations. ${ }^{29}$

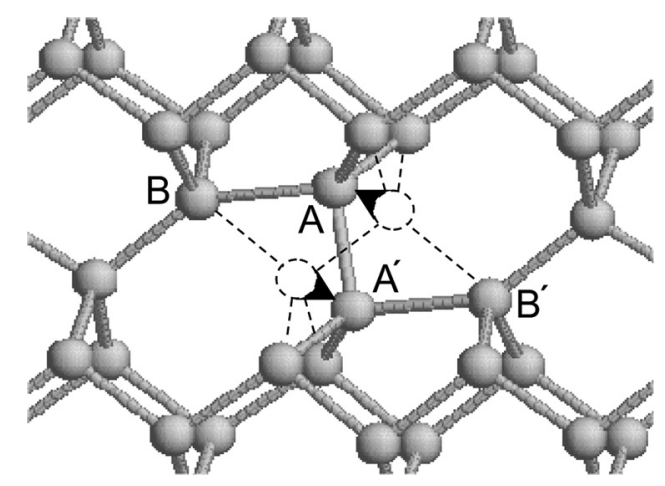

FIG. 2. Atomic structure of the bond defect. Dashed lines represent atoms and bonds in the perfect lattice. The bond defect is formed when atoms A and $\mathrm{A}^{\prime}$ move along the directions indicated by the arrows, switching bonds with atoms $\mathrm{B}$ and $\mathrm{B}^{\prime}$. Their reverse movement represents an elementary crystallization event.
In the experiments, the limiting surfaces of fins and nanowires are, at least, covered by a layer of native amorphous $\mathrm{SiO}_{2}{ }^{1,2,9}$ To simulate the influence of this surrounding $\mathrm{SiO}_{2}$ layer, which remains amorphous during the annealing step, we kept fixed the positions of atoms belonging to outer amorphous layers (to prevent its recrystallization). This approximation will reproduce the behavior of such amorphous $\mathrm{SiO}_{2}$ layers without the need of explicitly using a more complex potential describing the $\mathrm{Si}-\mathrm{O}$ interactions. For the sake of comparison and in order to exclude any possible artifact associated to the assumption of fixed amorphous interfaces surrounding the nanostructure, we have also carried out MD simulations of fins and nanowires with free surfaces.

In the case of the simulation of fin structures, periodic boundary conditions (PBC) are applied in the direction of the fin body (for nanowires no PBC are used). In both types of samples, top surfaces are left free (interfaces with vacuum) in order to accommodate any volume variation due to the different densities of amorphous and crystal Si. Samples are annealed at $1900 \mathrm{~K}$ for several nanoseconds. This temperature value is high enough to accelerate system dynamics but below the amorphous Si melting temperature predicted by T3. ${ }^{21}$ During crystal regrowth, energy is freed in the form of latent heat. While in the experiments, heat released during recrystallization dissipates throughout the sample and holder, in the simulation box this heat accumulates due to PBC and the lateral fixed interfaces with amorphous material. To avoid an artificial temperature increase in the simulation, atom velocities are rescaled every 1000 time steps to maintain $1900 \mathrm{~K}$ within the box. Equations of motion are integrated using the velocity-Verlet algorithm ${ }^{18}$ with a constant time step of $0.35 \mathrm{fs}$.

\section{RESULTS AND DISCUSSION}

As a first step, we tried to reproduce the experiments of Duffy et al. ${ }^{9}$ on sub-20 nm wide Si fins. They fabricated fin structures on (100) silicon-on-insulator wafers, patterned in the $\langle 110\rangle$ direction. After an implantation step, the fin body was fully amorphized, only the bottom portion remained crystalline (see Fig. 1). They carried out a subsequent annealing step at $1050{ }^{\circ} \mathrm{C}$. During annealing, the bottom crystalline part acted as a seed for recrystallization, but soon $\{111\}$ stacking faults started forming at the sides, as it was shown by crosssectional transmission electron microscopy images. Beyond them, polycrystalline silicon was generated. Thus, despite the presence of a lattice template at the bottom of the fin, recrystallization was highly defected, which produced an increase of the on-resistance of the final device. ${ }^{13,14}$ On the contrary, in bulk silicon systems, where regrowth occurs perpendicular instead of parallel to the surfaces/interfaces, recrystallization is almost perfect. ${ }^{7}$

Figure 3 shows several snapshots taken during a MD simulation of a $10 \mathrm{~nm}$ wide Si fin annealing surrounded by amorphous material. The initial configuration is shown in Fig. 3(a). It contains 84100 atoms and it is $11.2 \mathrm{~nm}$ thick. Crystal orientations are the same as in the previously described experiment. Regrowth proceeds from the bottom crystal seed but at the lateral interfaces crystallization is 
(a) $t=0$

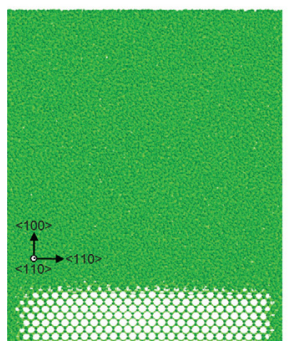

(b) $t=2.5 \mathrm{~ns}$

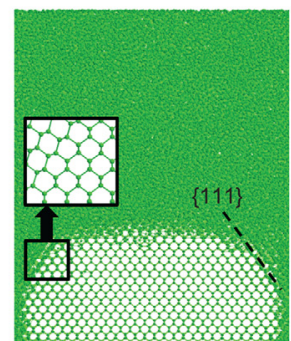

(c) t $=25 \mathrm{~ns}$

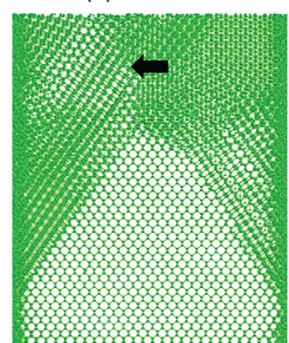

(d) Free surfaces

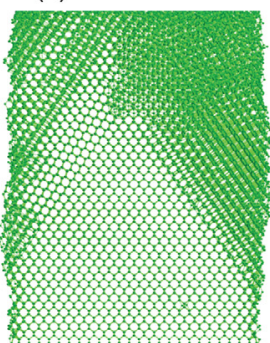

FIG. 3. (Color online) Several snapshots taken during a MD annealing at $1900 \mathrm{~K}$ of a $10 \mathrm{~nm}$ wide fin structure: (a) Initial configuration, (b) at $2.5 \mathrm{~ns}$, and (c) final configuration at $25 \mathrm{~ns}$ (arrow indicates the formation of poly-crystallites). Insert in (b) illustrates the formation of $\{111\}$ twin boundaries. (d) shows the final configuration of the annealing of a $10 \mathrm{~nm}$ wide fin surrounded by free surfaces. hindered, as it can be seen in Fig. 3(b). Meanwhile, the recrystallization front in the center of the fin advances in the $\langle 100\rangle$ direction. The influence of the regrowth retardation due to the presence of lateral interfaces propagates diagonally, which makes the regrowth front to adopt the typical form of an arrow tip limited by $\{111\}$ planes. Then, $\{111\}$ stacking faults start generating which retards further crystallization along the regrowth front. Spontaneous nucleation of crystal grains with random orientations occurs in the amorphous region beyond such stacking faults, which produces poly-crystalline silicon as observed in Fig. 3(c). The coincidence with experimental results of Duffy et $a l^{9}$ is striking, which indicates that our MD simulations adequately reproduce the physics involved in the fin regrowth process.

Analogous results are obtained when simulating the regrowth of a fin structure surrounded by free surfaces instead of amorphous material, as shown in Fig. 3(d). This suggests that the crystallization hindering at the lateral surfaces/interfaces is due to the lack of crystal periodicity on the sides that acts as a template for atomic rearrangement during solid phase epitaxial regrowth, independently of this being a consequence of having a free surface or an interface with amorphous material. For the sake of simplicity, from now on, we will only show our simulation results on the regrowth of $\mathrm{Si}$ nanostructures surrounded by amorphous material, since they are the same as when considering free surfaces instead.

In order to explain the reason behind the regrowth hindering at the lateral surfaces/interfaces, we have carried out MD simulations of the mean recombination lifetime of bond defects in the bulk and in the vicinity of a/c interfaces and free surfaces (simulation details are described elsewhere. ${ }^{30}$ ) As we have pointed out previously, the bond defect is the basic building block of the amorphous phase. Accumulation of bond defects in a perfect crystal lattice drives its amorphization. ${ }^{22,27}$ On the contrary, the recombination of a bond defect

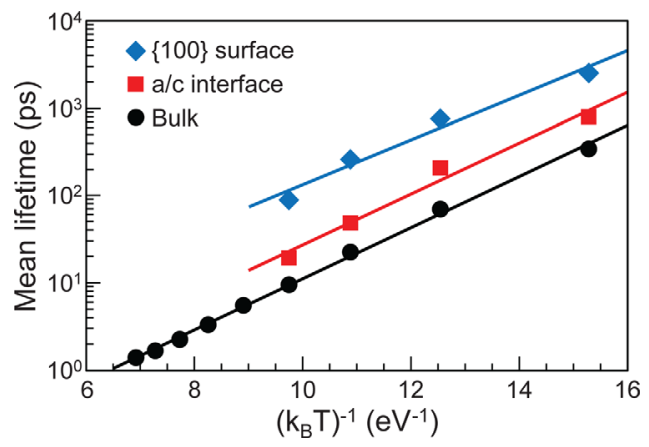

FIG. 4. (Color online) Arrhenius plot of average lifetimes of bond defects in the bulk, and when they are close to a/c interfaces and $\{110\}$ surfaces. leading to the recovery of the perfect crystal lattice can be considered as the basic recrystallization event (see Fig. 2). Figure 4 shows an Arrhenius plot of the mean recombination lifetimes of bond defects when they are in bulk, close to an $\mathrm{a} / \mathrm{c}$ interface, and close to a $\{100\}$ free surface. As it can be seen, lifetimes of bond defects close to surfaces and a/c interfaces are between one and two orders of magnitude longer compared to bond defects in bulk. Therefore, damage is much more stable near surfaces and a/c interfaces, which explains why crystallization is hindered precisely in the sides of fins shown in Fig. 3.

Another contribution to the defective regrowth is associated to the crystal orientation. It is well known that regrowth along the $\langle 111\rangle$ direction is slower that in $\langle 100\rangle$ and shows frequent defects in the stacking sequence. ${ }^{31,32}$ This is due to the fact that atoms in the $\{111\}$ a/c interface have only one bond with the crystalline phase (see Fig. 5(a)). Rotation around that bond can produce in some atomic layers further crystallization in the wurtzite structure (where bond angles
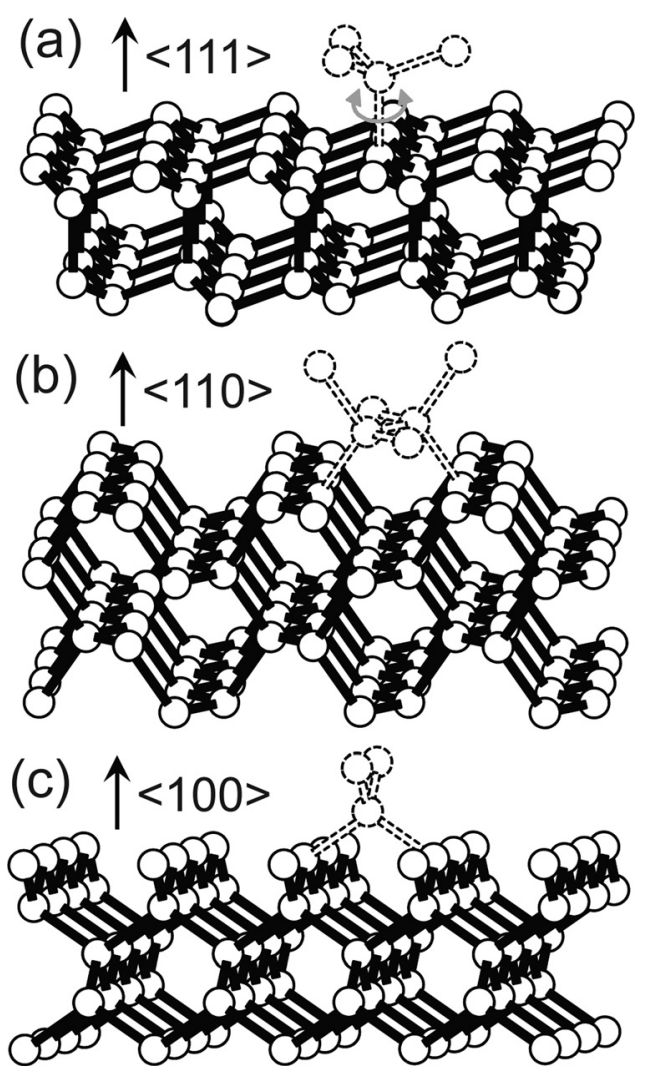

FIG. 5. Ball and stick representation indicating how atoms in the a/c interface have to rearrange (dashed lines) for crystal regrowth to proceed in: (a) the $\langle 111\rangle$ direction, (b) the $\langle 110\rangle$ direction, and (c) the $\langle 100\rangle$ direction. 
(a) $t=0$

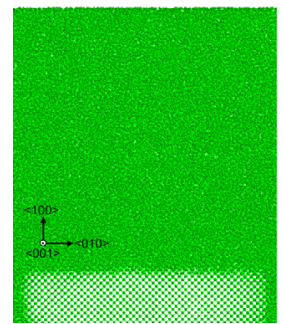

(b) $t=2.5 \mathrm{n}$

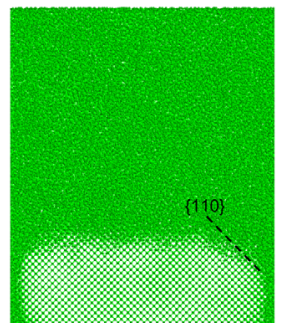

FIG. 6. (Color online) Several snapshots taken during a MD annealing at $1900 \mathrm{~K}$ of a $10 \mathrm{~nm}$ wide fin structure aligned along the $\langle 100\rangle$ direction: (a) Initial configuration, (b) at $2.5 \mathrm{~ns}$, and (c) final configuration at $25 \mathrm{~ns}$.

are the same as in the diamond structure), which leaves behind a twin boundary (shown in the insert of Fig. 3(b)). This generates $\{111\}$ stacking faults that retard further crystallization. In the particular orientation of the fin body considered in Fig. 3 and in the experiment by Duffy et al., recrystallization fronts, retarded at the lateral interfaces but continuing in the fin body center, catch up with $\{111\}$ planes. Due to the consequent recrystallization slowdown, spontaneous nucleation of crystal grains in random orientations in the fully amorphous region beyond the staking faults becomes the fastest regrowth mechanism, producing the formation of polysilicon. ${ }^{33}$ In planar devices, recrystallization is not hindered and crystal grains have not enough time for nucleating before full regrowth occurs.

Consequently, defected regrowth is due to the recrystallization slowdown close to the lateral surfaces/interfaces, and the recrystallization front being limited by $\{111\}$ planes. Recrystallization quality could be then improved if the fin is aligned along a different crystal direction. In Fig. 6(a), we show several MD snapshots taken during the annealing of a $10 \mathrm{~nm}$ wide $\mathrm{Si}$ fin, now aligned along the $\langle 100\rangle$ direction. The box contains 88200 atoms and it is $11.5 \mathrm{~nm}$ thick. As it can be seen, recrystallization is improved with respect to the $\langle 110\rangle$ oriented fin. Now, the lateral interface influence propagates diagonally along $\{110\}$ planes. Recrystallization along (a) $t=0$

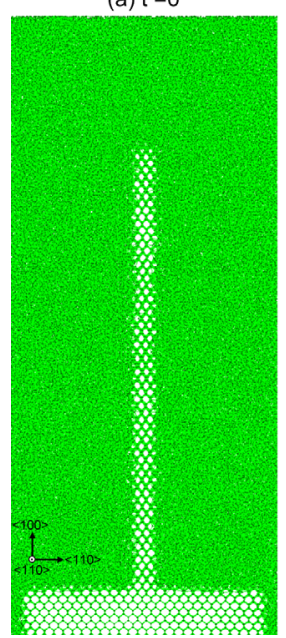

(b) t $=5 \mathrm{~ns}$

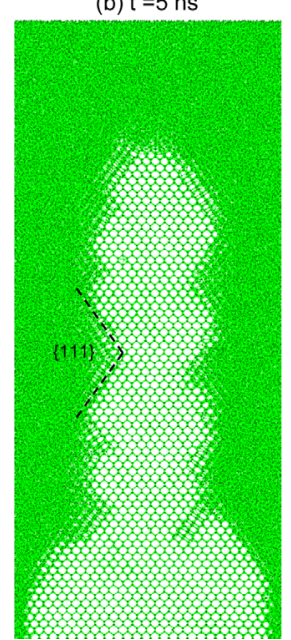

(c) t $=35 \mathrm{~ns}$

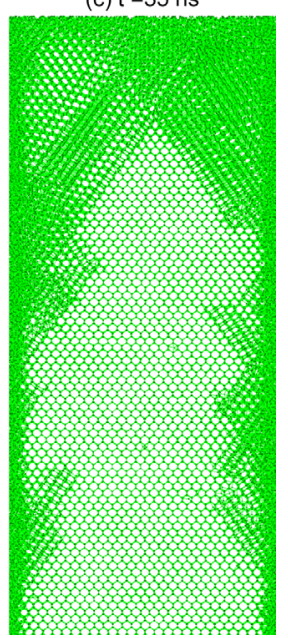

FIG. 7. (Color online) Several snapshots taken during a MD annealing at $1900 \mathrm{~K}$ of a $10 \mathrm{~nm}$ wide fin structure aligned along the $\langle 110\rangle$ direction with a $1 \mathrm{~nm}$ crystalline seed in the middle of the body: (a) Initial configuration, (b) at $5 \mathrm{~ns}$, and (c) final configuration at $35 \mathrm{~ns}$. the $\langle 110\rangle$ direction is faster that in $\langle 111\rangle$ and does not show defects in the stacking sequence as frequently. ${ }^{31,32}$ Nevertheless, although the bottom a/c interface has advanced $6 \mathrm{nms}$ with respect to its original position, some defective structures do form beyond that distance. This is because, in order to advance the $\mathrm{a} / \mathrm{c}$ interface along the diagonal $\langle 110\rangle$ direction, two atoms in the amorphous phase have to bond together and with two atoms in the crystal phase as indicated in Fig. 5(b). A little mismatch in the location of these two new crystalline atoms with respect to the perfect crystal positions can nucleate a defect in the a/c interface. Sometimes, these defects are small in size, are encompassed by perfect crystal, and disappear with further annealing. But occasionally, they accumulate and generate stacking faults along $\{110\}$ planes, as it can be seen in Fig. 6(c).

Another alternative to improve recrystallization consists of leaving a crystalline seed in the middle of the fin. This would be possible by selecting appropriate implant parameters in the experiment. Figure 7 shows several snapshots taken during the MD simulation of the annealing of a $10 \mathrm{~nm}$ wide $\mathrm{Si}$ fin aligned along the $\langle 110\rangle$ direction, with a thin crystalline seed (just $1 \mathrm{~nm}$ thick) in the middle of the fin body. The initial configuration is shown in Fig. 7(a). It contains 168200 atoms and it is $11.2 \mathrm{~nm}$ thick. Now, there are two recrystallization fronts, one advancing parallel to the lateral interfaces in the $\langle 100\rangle$ direction and another one perpendicular to lateral interfaces in the $\langle 110\rangle$ direction. As it can be seen, even though regrowth is again limited by $\{111\}$ planes (dashed lines in Fig. 7(b)), recrystallization quality is much improved with respect to MD simulations shown in Fig. 3.

During an amorphizing implant, a high number of Si selfinterstitials are injected beyond the a/c interface. ${ }^{5,6}$ This means that in the experiment, even if you adjust the implantation parameters to leave a crystal seed in the middle of the fin body, it will be populated by a large number of $\mathrm{Si}$ selfinterstitial defects, which could influence the quality of regrowth. In order to reproduce more accurately the experimental situation, we have repeated the previously described MD simulation but introducing Si self-interstitial atoms within the initial crystal seed to a concentration of $4 \times 10^{21} \mathrm{~cm}^{-3}$ (of the order of residual defect concentrations at typical implant doses). ${ }^{5}$ These defects were introduced randomly in interstitial positions along the middle crystal seed. Simulation results indicate that even if the center of the fin is highly damaged (but not fully amorphized), the lateral regrowth is good enough, as it is shown in Fig. 8. Most of the introduced Si self-interstitial defects diffuse to the a/c interfaces and then are swept to the sides as the recrystallization fronts advance (they never come back to the crystal seed).

Recrystallization is improved further if the fin is aligned along the $\langle 100\rangle$ direction and contains a crystalline seed parallel to the lateral interfaces. Again, we simulated the annealing of a $10 \mathrm{~nm}$ wide $\mathrm{Si}$ fin with a $1 \mathrm{~nm}$ crystalline seed in the middle of the fin body. Several snapshots taken during the simulation are shown in Fig. 9. The initial configuration contains 176400 atoms and it is $11.5 \mathrm{~nm}$ thick. Now both crystallization fronts are $\{100\}$ planes. Growth along the $\langle 100\rangle$ direction is fastest ${ }^{32}$ and it shows few defects in the stacking sequence because the basic crystallization event involves only one atom 

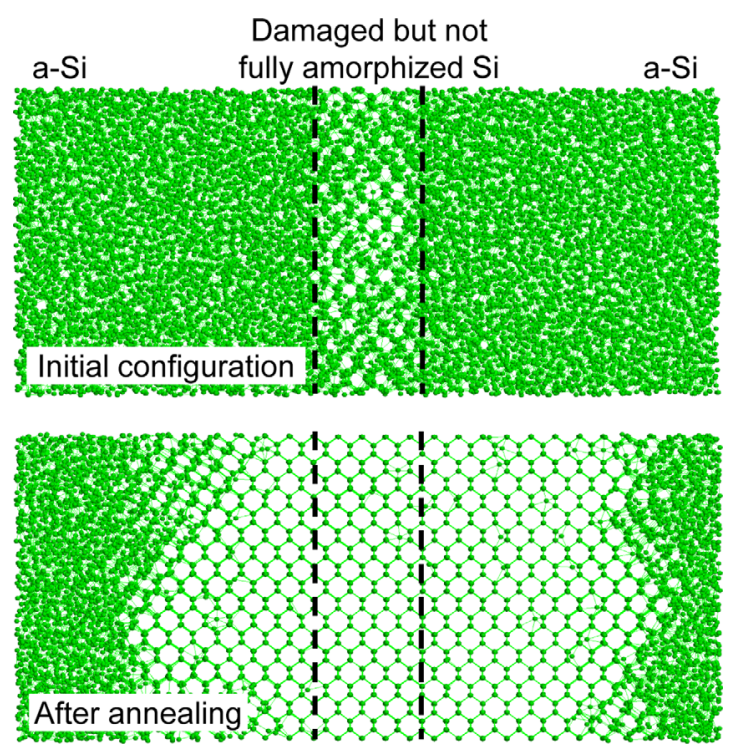

FIG. 8. (Color online) Snapshots taken during annealing at $1900 \mathrm{~K}$ showing a section of a FinFET with two lateral amorphous regions and a highly damaged but not fully amorphized region in the center. Orientations are the same as in Fig. 7.

that forms two bonds with the underlying crystal phase (see Fig. 5(c)), so no rotation or mismatch is possible in this case. The two advancing interfaces promote perfect recrystallization even though sometimes the regrowth front catches up with $\{110\}$ planes (see Fig. 9(b)), again due to the presence of lateral interfaces. At the end of the annealing, the full body of the fin has recrystallized almost perfectly up to the tip of the initial crystal seed, as shown in Fig. 9(c).

We have carried out similar MD simulations in nanowire structures. In particular, we have focused on $\langle 100\rangle$ oriented nanowires, since that is the crystal orientation that has shown better regrowth behavior in the case of fins. We have annealed a $10 \times 10 \mathrm{~nm}$ nanowire, surrounded by amorphous material in both lateral directions (no PBC are used in this case), at a temperature of $1900 \mathrm{~K}$. The total number of atoms in the simulation is 96000 . Figure 10(a) shows snapshots taken at the beginning and the end of the nanowire annealing simulation. To clearly see the inner structure of the nanowire, only atoms within a $2 \mathrm{~nm}$ slice in its center are shown. (a) $t=0$

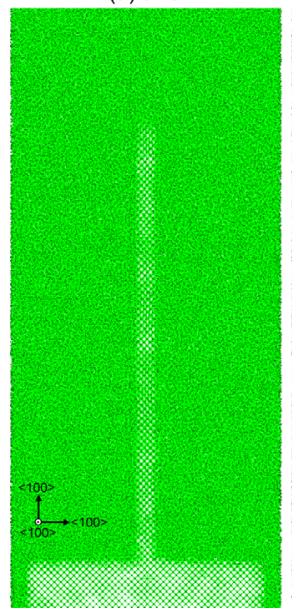

(b) $t=5 n s$
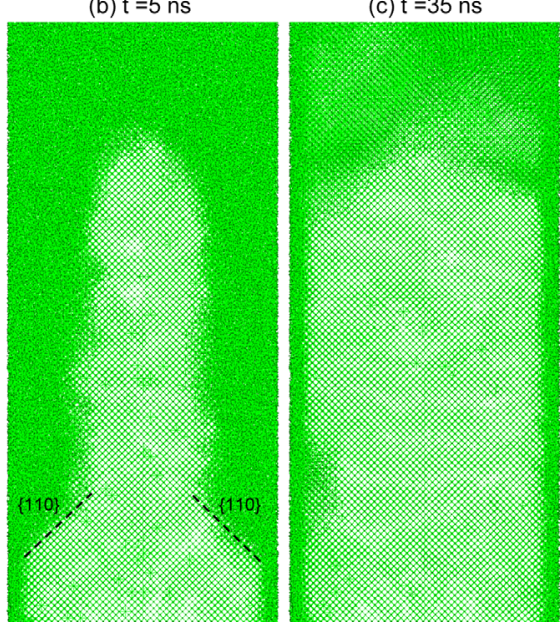

FIG. 9. (Color online) Several snapshots taken during a MD annealing at $1900 \mathrm{~K}$ of a $10 \mathrm{~nm}$ wide fin structure aligned along the $\langle 100\rangle$ direction with a $1 \mathrm{~nm}$ crystalline seed in the middle of the body: (a) Initial configuration, (b) at $5 \mathrm{~ns}$, and (c) final configuration at $35 \mathrm{~ns}$.

As it can be seen, the final structure corresponds to a polycrystalline material. In the fin case, we observed that regrowth can be improved if a crystalline seed is left in the middle of the body. To see if that is also the case for nanowires, we repeated the annealing simulation with a $1 \times 1 \mathrm{~nm}$ section crystal seed in the nanowire center. Initial and final configurations are shown in Fig. 10(b). In this last case, recrystallization is faster but, unlike in the fin case, the final material is also polycrystalline. Nevertheless, polycrystals are bigger and less misoriented with respect to the $\langle 100\rangle$ direction than in the first case.

The different behavior of nanowires and fins with respect to regrowth is a consequence of how the crystal phase is confined within the amorphous material. In Fig. 11, we show several side views taken during the annealing of the seeded nanowire and fin of Fig. 9. Atoms displayed in the figure were taken from a $2 \mathrm{~nm}$ thick slice $10 \mathrm{~nm}$ above the bottom crystal seed. As can be seen, in the fin case the inner crystal seed is confined in only one direction (in the other one, in which the fin is oriented, there is no confinement, and PBC conditions are used). The a/c interfaces (oriented along the $\langle 100\rangle$ direction) remain almost planar along regrowth, and at the end,

(a)

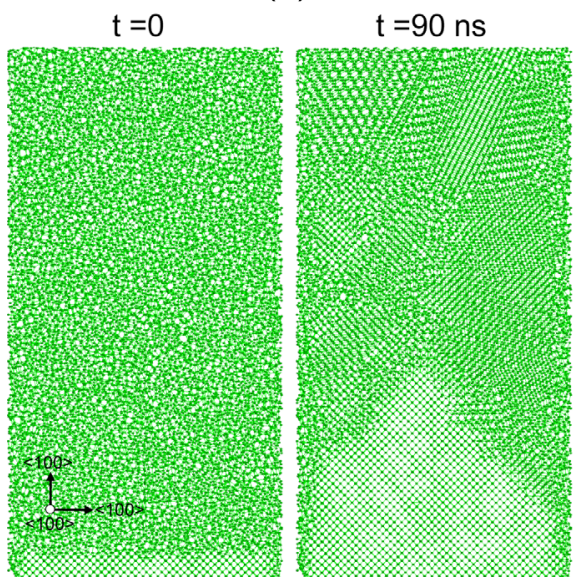

(b)

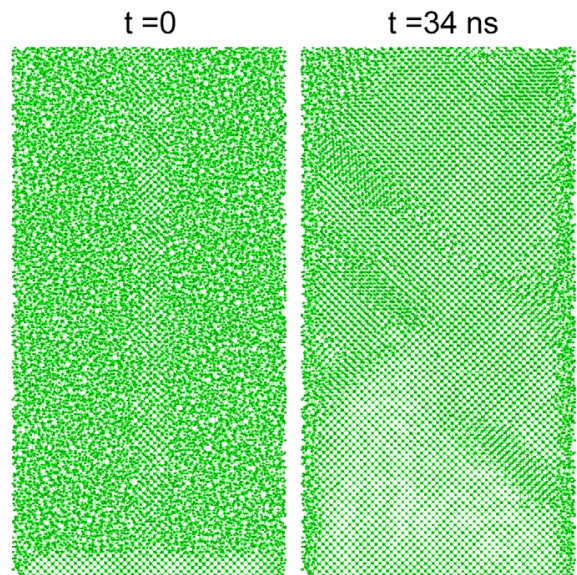

FIG. 10. (Color online) Initial and post-anneal configurations of $\mathrm{Si}$ nanowires surrounded by amorphous material in both lateral directions, (a) without and (b) with a crystal seed in its center. For the sake of clarity, only atoms within a $2 \mathrm{~nm}$ slice in the center of the nanowire are shown. 

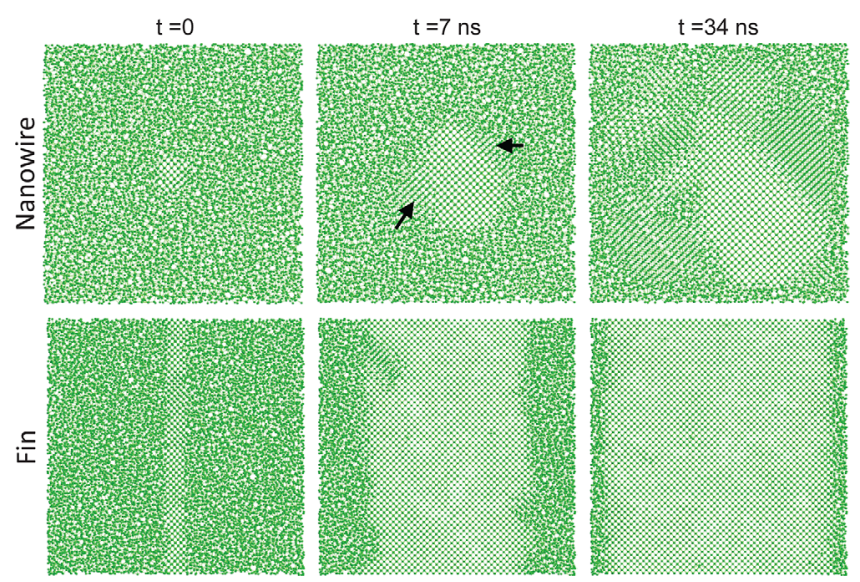

FIG. 11. (Color online) Side views taken during the annealing of the seeded nanowire and fin oriented along the $\langle 100\rangle$ direction. Atoms shown have been taken from a $2 \mathrm{~nm}$ thick slice $10 \mathrm{~nm}$ above the bottom crystal seed. Arrows indicate the staking faults that start forming in the nanowire case.

recrystallization is perfect but for some point defects. However, in the nanowire case, the inner crystal seed is confined within the amorphous phase in the two lateral dimensions at the same time. This retards regrowth with respect to the fin case. Soon recrystallization fronts catch with the $\langle 110\rangle$ direction and stacking faults start forming, which promotes the generation of polycrystalline material.

\section{CONCLUSIONS}

We have used the MD simulation technique to study regrowth issues of nanometric $\mathrm{Si} 3 \mathrm{D}$ structures, such as FinFETs and nanowires. We have shown that annealing, needed to recover the crystalline structure of the device body after implantation, produces imperfect regrowth in agreement with experimental observations. Our results indicate that this defected crystallization is due to the presence of lateral free surfaces or interfaces with amorphous material. They slow down recrystallization with respect to bulk, so regrowth proceeds at different velocities depending on the distance to the lateral surfaces/interfaces. Consequently, the recrystallization front adopts the form of an arrow tip. Regrowth then proceeds depending on the particular orientation of the new a/c interfaces. In the particular case of $\langle 110\rangle$ oriented fins, the a/c interfaces on the arrow tip are aligned along the $\langle 111\rangle$ direction, which produces frequent twining during further regrowth. This retards overall recrystallization so nucleation of crystal grains at random orientations beyond the a/c interfaces start occurring, producing the formation of polysilicon.

Based on our simulations, we have proposed technological alternatives to overcome this defected regrowth: (a) to modify the implant parameters so that a crystal seed remains in the device body center and (b) to change the device body orientation from $\langle 110\rangle$ to $\langle 100\rangle$. In both cases, we have shown that the quality of the recrystallization is improved. However, for narrow nanowires, perfect regrowth is not possible due to the presence of surrounding surfaces/interfaces in both lateral directions.

\section{ACKNOWLEDGMENTS}

This work has been partially funded by the Spanish Government under Project No. TEC2008-06069.

${ }^{1}$ J. L. Liu, Y. Lu, Y. Shi, S. L. Gu, R. L. Jiang, F. Wang, and Y. D. Zheng, Appl. Phys. A 66, 539 (1998).

${ }^{2}$ Y. Cui, Z. Zhong, D. Wang, W. U. Wang, and C. M. Lieber, Nano Lett. 3, 149 (1982).

${ }^{3}$ See http://www.itrs.net/for International Technology Roadmap for Semiconductors.

${ }^{4}$ C. Ronning, C. Borschel, S. Geburt, and R. Niepelt, Mater. Sci. Eng. R. 70, 30 (2010).

${ }^{5}$ L. Pelaz, M. Aboy, P. López, and L. A. Marqués, J. Vac. Sci. Technol. B 24, 2432 (2006)

${ }^{6}$ P. F. Fazzini, F. Cristiano, C. Dupré, S. Paul, T. Ernst, H. Kheyrandish, K. K. Bourdelle, and W. Lerch, Mater. Sci. Eng. B 154-155, 256 (2008).

${ }^{7}$ E. Lampin and C. Krzeminski, J. Appl. Phys. 106, 063519 (2009).

${ }^{8}$ K. L. Saenger, J. P. de Souza, K. E. Fogel, J. A. Ott, C. Y. Sung, D. K. Sadana, and H. Yin, J. Appl. Phys. 101, 024908 (2007).

${ }^{9}$ R. Duffy, M. van Dal, B. Pawlak, M. Kaiser, R. Weemaes, B. Degroote, E. Lunnen, and E. Al-tamirano, Appl. Phys. Lett. 90, 241912 (2007).

${ }^{10}$ I. Ferain, R. Duffy, N. Collaert, M. J. H. van Dal, B. J. Pawlak, B. O’Sullivan, L. Witters, R. Rooyackers, T. Conard, M. Popovici, S. van Elshocht, M. Kaiser, R. G. R. Weemaes, J. Swerts, M. Jurczak, R. J. P. Lander, and K. De Meyer, Solid-State Electron. 53, 760 (2009).

${ }^{11}$ H. Ishiwara, H. Yamamoto, S. Furukawa, M. Tamura, and T. Tokuyama, Appl. Phys. Lett. 43, 1028 (1983).

${ }^{12}$ Y. Kunni, T. Michibaru, and K. Kajiyama, J. Appl. Phys. 56, 279 (1984).

${ }^{13}$ M. van Dal, N. Collaert, G. Doornbos, G. Vellianitis, G. Curatola, B. Pawlak, R. Duffy, C. Jonville, B. Degroote, E. Altamirano, E. Kunnen, M. Demand, S. Beckx, T. Vandeweyer, C. Delvaux, F. Leys, A. Hikavyy, R. Rooyackers, M. Kaiser, R. G. R. Weemaes, S. Biesemans, M. Jurczak, K. Anil, L. Witters, and R. J. P. Lander, in Dig. Tech. Pap. - Symp. VLSI Technol. 2007, 110.

${ }^{14}$ T. Merelle, G. Curatola, G. Nackaerts, A. Collaert, M. J. H. van Dal, G. Doornbos, T. S. Doorn, P. Christie, G. Vellianitis, B. Duriez, R. Duffy, B. J. Pawlak, F. C. Voogt, R. Rooyackers, L. Witters, M. Jurczak, and R. J. P. Lander, in Tech. Dig. - Int. Electron Devices Meet. 2008, 241.

${ }^{15}$ L. Pelaz, R. Duffy, M. Aboy, L. A. Marqués, P. López, I. Santos, B. J. Pawlak, M. J. H. van Dal, B. Duriez, T. Merelle, G. Doornbos, N. Collaert, L. Witters, R. Rooyackers, W. Vandervorst, M. Jurczak, M. Kaiser, R. G. R. Weemaes, J. G. M. van Berkum, P. Breimer, and R. J. P. Lander, Tech. Dig. - Int. Electron Devices Meet. 2008, 535.

${ }^{16}$ E. Lampin and C. Krzeminski, J. Appl. Phys. 109, 123509 (2011).

${ }^{17}$ I. Martín-Bragado, Appl. Phys. Lett. 98, 233109 (2011).

${ }^{18}$ J. M. Haile, Molecular Dynamics Simulations: Elementary Methods (Wiley, New York, 1992).

${ }^{19}$ J. Tersoff, Phys. Rev. B 39, 5566 (1989).

${ }^{20}$ M. Ishimaru, S. Munetoh, and T. Motooka, Phys. Rev. B 56, 15133 (1997).

${ }^{21}$ L. A. Marqués, L. Pelaz, M. Aboy, and J. Barbolla, Nucl. Instrum. Methods Phys. Res. B 216, 57 (2004).

${ }^{22}$ L. A. Marqués, L. Pelaz, J. Hernández, J. Barbolla, and G. H. Gilmer, Phys. Rev. B 64, 45214 (2001).

${ }^{23}$ J. W. Mayer and S. S. Lau, Electronics Materials Science for Integrated Circuits in Si and GaAs (MacMillan, New York, 1990).

${ }^{24}$ L. J. Porter, S. Yip, M. Yamaguchi, H. Kaburaki, and M. Tang, J. Appl. Phys. 81, 96 (1997).

${ }^{25}$ M. Tang, L. Colombo, J. Zhu, and T. D. de la Rubia, Phys. Rev. B 55, 14279 (1997).

${ }^{26}$ F. Cargnoni, C. Gatti, and L. Colombo, Phys. Rev. B 57, 170 (1998).

${ }^{27}$ L. A. Marqués, L. Pelaz, M. Aboy, L. Enríquez, and J. Barbolla, Phys. Rev. Lett. 91, 135504 (2003).

${ }^{28}$ F. Wooten, K. Winer, and D. Weaire, Phys. Rev. Lett. 54, 1392 (1985).

${ }^{29}$ L. Pelaz, L. A. Marqués, and J. Barbolla, J. Appl. Phys. 96, 5947 (2004).

${ }^{30}$ I. Santos, Ph.D. thesis, Univ. de Valladolid, Spain, 2010.

${ }^{31}$ L. Csepregi, J. W. Mayer, and T. W. Sigmon, Appl. Phys. Lett. 29, 92 (1976).

${ }^{32}$ B. Drosd and J. Washburn, J. Appl. Phys. 53, 397 (1982).

${ }^{33}$ K. F. Kelton, A. L. Greer, and C. V. Thompson, J. Chem. Phys. 79, 6261 (1983). 